\title{
Circulating CD15+LOX-1+ PMN-MDSCs are a potential biomarker for the early diagnosis of non-small cell lung cancer
}

\author{
Xinyu Tian ${ }^{1}$, Ting Wang ${ }^{2}$, Qisi Zheng ${ }^{1}$, Yue Tao $^{1}$, Lei Dai ${ }^{1}$, and Han Shen ${ }^{1}$ \\ ${ }^{1}$ Nanjing Drum Tower Hospital \\ ${ }^{2}$ Jiangsu Cancer Hospital
}

November 29, 2020

\begin{abstract}
Aims: Non-small-cell lung cancer (NSCLC) is the most common clinical lung cancer. Polymorphonuclear-myeloid derived suppressor cells (PMN-MDSCs), which are the major population of MDSCs, are involved in NSCLC progression. Recently, it was found that lectin-type oxidized LDL receptor 1 (LOX-1) could identify humsn PMN-MDSCs. However, the role of CD15+LOX-1+ PMN-MDSCs in NSCLC early diagnosis has not been revealed. Here, we tried to confirm the application of the newly-identified CD15+LOX-1+ PMN-MDSCs in the early diagnosis of NSCLC. Methods: Flow cytometry (FCM) was used to detect the proportion of CD15+LOX-1+ PMN-MDSCs in the peripheral blood (PB) of healthy controls (HC) and NSCLC patients. The correlation of CD15+LOX-1+ PMN-MDSC frequency with levels of cytokeratin 19-fragments (CYFRA21-1), carcinoembryonic antigen (CEA), and carbohydrate antigen 125 (CA125) was analyzed. Receiver operating characteristic (ROC) curve was used to estimate the diagnostic efficacy of CD15+LOX-1+ PMN-MDSCs for NSCLC. Additionally, the association of CD15+LOX-1+ PMN-MDSC frequency with NSCLC prognosis/recurrence after surgery was explored. Results: The proportion of CD15+LOX-1+ PMN-MDSCs increased in PB of NSCLC patients. CD15+LOX-1+ PMN-MDSC proportion was positively correlated with levels of CEA and CYFRA21-1. The area under the ROC curve (AUC) of PMN-MDSC percentage was higher than CYFRA21-1, CEA and CA125. The proportion of CD15+LOX-1+ PMN-MDSCs decreased in patients after surgery. The frequency of CD15+LOX-1+ PMN-MDSCs was lower in NSCLC patients without recurrence compared to those with recurrence after surgery. Conclusions: Circulating CD15+LOX-1+ PMN-MDSCs are a potential diagnostic marker for NSCLC, and are associated with NSCLC prognosis and recurrence after surgery.
\end{abstract}

Circulating CD15 ${ }^{+}$LOX-1 ${ }^{+}$PMN-MDSCs are a potential biomarker for the early diagnosis of non-small cell lung cancer

Running title: CD15 ${ }^{+}$LOX-1+ ${ }^{+}$PMN-MDSCs are a biomarker of NSCLC

Xinyu Tian ${ }^{1 \#^{*}}$, Ting Wang ${ }^{2 \#}$, Qisi Zheng ${ }^{1 \#}$, Yue Tao ${ }^{1}$, Lei Dai ${ }^{1}$, and Han Shen ${ }^{1}$

Department of Laboratory Medicine, Nanjing Drum Tower Hospital, Nanjing University Medical School, Nanjing, Jiangsu, China

Department of Laboratory Medicine, Jiangsu Cancer Hospital \& Jiangsu Institute of Cancer Research \& The Affiliated Cancer Hospital of Nanjing Medical University, Nanjing, Jiangsu, China

*Correspondence:

Xinyu Tian, Department of Laboratory Medicine, Nanjing Drum Tower Hospital, 321 Zhongshan Road, Nanjing 210008, China. Tel.: +86025 83106666; E-mail: 1000004433@ujs.edu.cn

\# The three authors contributed equally.

\section{DISCLOSURES}


For each author there were no conflicts of interests, including all relevant financial interest in any company or institution that might benefit from the publication.

\title{
ACKNOWLEDGEMENTS
}

This work was supported by Jiangsu Planned Projects for Postdoctoral Research Funds (2018K253C), China's Post-doctoral Science Fund (2018ZM642225), and Funds of Nanjing Medical University Affiliated Cancer Hospital (ZM201704).

Circulating CD15 ${ }^{+} \mathrm{LOX}-1^{+}$PMN-MDSCs are a potential biomarker for the early diagnosis of non-small cell lung cancer

\begin{abstract}
Aims: Non-small-cell lung cancer (NSCLC) is the most common clinical lung cancer. Polymorphonuclearmyeloid derived suppressor cells (PMN-MDSCs), which are the major population of MDSCs, are involved in NSCLC progression. Recently, it was found that lectin-type oxidized LDL receptor 1 (LOX-1) could identify humsn PMN-MDSCs. However, the role of CD15+LOX-1+ PMN-MDSCs in NSCLC early diagnosis has not been revealed. Here, we tried to confirm the application of the newly-identified CD15 ${ }^{+} \mathrm{LOX}-1^{+}$ PMN-MDSCs in the early diagnosis of NSCLC.
\end{abstract}

Methods: Flow cytometry (FCM) was used to detect the proportion of CD15+LOX-1+ PMN-MDSCs in the peripheral blood (PB) of healthy controls (HC) and NSCLC patients. The correlation of CD15 ${ }^{+} \mathrm{LOX}-$ $1^{+}$PMN-MDSC frequency with levels of cytokeratin 19-fragments (CYFRA21-1), carcinoembryonic antigen (CEA), and carbohydrate antigen 125 (CA125) was analyzed. Receiver operating characteristic (ROC) curve was used to estimate the diagnostic efficacy of CD $15^{+} \mathrm{LOX}-1^{+}$PMN-MDSCs for NSCLC. Additionally, the association of $\mathrm{CD} 15^{+} \mathrm{LOX}-1^{+}$PMN-MDSC frequency with NSCLC prognosis/recurrence after surgery was explored.

Results: The proportion of CD15 ${ }^{+}$LOX-1 $1^{+}$PMN-MDSCs increased in PB of NSCLC patients. CD15 ${ }^{+}$LOX$1^{+}$PMN-MDSC proportion was positively correlated with levels of CEA and CYFRA21-1. The area under the ROC curve (AUC) of PMN-MDSC percentage was higher than CYFRA21-1, CEA and CA125. The proportion of CD15+LOX-1+ PMN-MDSCs decreased in patients after surgery. The frequency of CD15+LOX-1 ${ }^{+}$ PMN-MDSCs was lower in NSCLC patients without recurrence compared to those with recurrence after surgery.

Conclusions: Circulating CD15 ${ }^{+}$LOX $-1^{+}$PMN-MDSCs are a potential diagnostic marker for NSCLC, and are associated with NSCLC prognosis and recurrence after surgery.

KEYWORDS: Polymorphonuclear-myeloid derived suppressor cells, non-small cell lung cancer, biomarker, early diagnosis, recurrence after surgery

\section{WHAT IS KNOWN?}

PMN-MDSCs are involved in the progression of NSCLC by promoting tumor immune escape and metastasis.

There lack specific biomarkers for identifying PMN-MDSCs.

\section{WHAT IS NEW?}

1. $\mathrm{CD} 15^{+} \mathrm{LOX}-1^{+}$PMN-MDSCs are a potential biomarker for NSCLC early diagnosis.

2. CD15+LOX-1+ PMN-MDSCs are associated with NSCLC prognosis and recurrence after surgery.

\section{INTRODUCTION}

Lung cancer has become a challenging health problem worldwide. More than 1.0 million people die of the disease each year $[1,2]$. According to the histopathological classification, lung cancer can be divided into small cell lung cancer (SCLC) and NSCLC, in which NSCLC accounts for approximately $85 \%$ and is the most common type of clinical lung cancer. NSCLC can be subdivided into squamous cell carcinoma, 
adenocarcinoma and large cell carcinoma [3-5]. In spite of the development in lung cancer diagnosis and treatment, the overall survival of NSCLC still remains at a low rate [6-9]. Metastasis is identified as the main cause of lung cancer related mortalities after resection. It is confirmed that 30-55\% of NSCLC patients in early stage die from recurrent metastatic disease after surgery with curative intent, and only $3-10 \%$ of them can survive over 5 years with the treatment of standard adjuvant chemotherapy [10-12]. Immune checkpoint blockade that can promote the generation of antigen specific $\mathrm{T}$ cells is a newly defined immune adjuvant therapy $[13,14]$. However, most of early-stage NSCLC tumors are removed without neoadjuvant intervention in clinical practice $[11,15]$. Current biomarkers for NSCLC such as CYFRA21-1, CEA and CA125 are not specific and sensitive enough to reflect the NSCLC progression. Therefore, specific biomarkers are necessary for monitoring NSCLC development and developing targeted treatment approaches for NSCLC.

In the progression of lung cancer, there exist multiple immune cells infiltrating into tumor sites. Different types of these immune cells and their products determine the fate of tumor progression. Immune cells also play a major role in establishing metastasis of the primary tumor to various organs [16-19]. A protective immune response against tumor is often prevailed by the protumor response, and it eventually is the balance or the lack of it between these two processes that determine the fate of tumor growth and metastasis. MDSCs are one of the immuosuppressive populations, which are rapidly attracting attention in tumor biology [20]. MDSCs are a population of immature myeloid cells (IMCs) and mature myeloid cells with immune suppressive activity. Under physiological conditions, IMCs produced by bone marrow can differentiate into mature granulocytes, monocytes and dendritic cells (DCs). In pathological conditions, especially cancer, the differentiation of IMCs is inhibited by molecules released by tumor cells, stromal cells and activated immune cells, which leads to the accumulation of MDSCs [21-23]. In human and mice, PMN-MDSCs and monocytic MDSCs (M-MDSCs) are the two main subsets of MDSCs, in which PMN-MDSCs that account for $70-80 \%$ of total MDSCs are the prioritized population [24]. In tumor microenvironment (TME), PMN-MDSCs are the main subset of MDSCs that induce tumor immune escape. Besides PMN-MDSCs migrating from bone marrow to TME, M-MDSCs and mature granulocytes in TME can also convert into PMN-MDSCs to induce the immunosuppression more effectively [25]. PMN-MDSCs have previously been confirmed to be involved in the progression of NSCLC by promoting tumor immune escape and metastasis in both preclinical and clinical trails [26-28]. However, PMN-MDSCs, which were initially identified as a population of terminally differentiated neutrophils with immunosuppression, shared the similar morphology and phenotype with mature ones. Therefore, it was the suppressive activity, but not phenotype, was the most reliable approach to distinguish PMN-MDSCs from neutrophils [20]. Recently, it was confirmed that LOX-1 could be used to distinguish the population of PMN-MDSCs in cancer patients. It was found that the newly defined LOX-1 $1^{+} \mathrm{CD} 15^{+}$PMN-MDSCs enhanced immune suppression which promoted tumor progression [2932]. Thus, we attempted to ensure the application of CD $15^{+} \mathrm{LOX}-1^{+}$PMN-MDSCs in NSCLC diagnosis in present study, as well as identify their association with NSCLC prognosis and recurrence after surgery.

\section{MATERIALS AND METHODS}

\section{Patients and samples}

PB samples of primary NSCLC patients (Adenocarcinoma, $n=27$; Squamous cell lung cancer, $n=23$ ) and healthy donors $(n=50)$ were obtained from Nanjing Drum Tower Hospital from April 2018 to December 2019. Cancer cases were confirmed by pathological diagnosis. According to the results of surgery, cancer staging was determined according to the 6th edition of the American Joint Cancer Commission's tumor-node-metastasis (TNM) staging system. All NSCLC patients were diagnosed for the first time and had not received any treatment before. Detailed information of NSCLC patients were listed in Table 1. Collected samples were firstly subjected to centrifuge at $20^{\circ} \mathrm{C}, 2000 \mathrm{rpm}$ for $5 \mathrm{~min}$ to separate the cells from plasma. ACK buffer was used to lyse red blood cells (RBCs), and the remaining cells were used for following experiments. To confirm the potential application of PMN-MDSC frequency in NSCLC diagnosis, we also collected paired PB samples from NSCLC patients at 3 months after surgical resection. Meanwhile, PB samples of NSCLC patients with or without recurrence after surgery were collected to identify the association of PMN-MDSC proportion with NSCLC recurrence $(\mathrm{n}=50)$. All samples were obtained in accordance with the approval of the Ethics 
Committee of Nanjing Drum Tower Hospital. All experiments were undertaken with the understanding and written consent of each subject.

\section{FCM}

To detect the proportion of CD15+LOX-1+ PMN-MDSCs in PB of NSCLC patients and HC, ACK buffer was used to lyse RBCs, and the remaining cells were stained with PE/Cy5.5-anti-human-CD11b (eBioscience, $0.25 \mu \mathrm{g} / 1 \times 10^{6}$ cells), APC-anti-human-CD33 (eBioscience, $0.25 \mu \mathrm{g} / 1 \times 10^{6}$ cells), PE-anti-human-LOX1 (eBioscience, $0.25 \mu \mathrm{g} / 1 \times 10^{6}$ cells) and FITC-anti-human-CD15 mAbs (eBioscience, $0.25 \mu \mathrm{g} / 1 \times 10^{6}$ cells) at $4^{\circ} \mathrm{C}$ for 30 min. FCM was performed using a FACSCalibur flow cytometer (Becton Dickinson). Data were analyzed by FlowJo software.

\section{Reverse transcript PCR and quantitative real-time PCR}

The RNA isolation, reverse transcript PCR and quantitative real-time PCR (qRT-PCR) were performed as described previously. The sequences for the primers used are: $\beta$-Actin: Forward: 5GAGTGTGGAGACCATCAAGGA-3, Reverse: 5-TGTATTGCTTTGCGTTGGAC-3; ARG1: Forward: 5CCTTTGCTGACATCCCTAAT-3, Reverse: 5-GATTCTTCCGTTCTTCTTGACT-3.

\section{Detection of tumor markers}

Levels of CEA, CA125, and CYFRA21-1 in serum of NSCLC patients were measured by full automatic electrochemiluminescent immunoassay (Roche, Switzerland). The upper limits of detection results were 3.5 $\mathrm{ng} / \mathrm{mL}, 35 \mathrm{U} / \mathrm{mL}$, and $3.3 \mathrm{ng} / \mathrm{mL}$, respectively.

\section{Graphing and statistical analysis}

Data were presented as Mean \pm SD. GraphPad Prism 5.0 (GraphPad, San Diego, CA) was used to generate bar graphs and ROC curves. The statistical significance of differences between groups was determined by the Student's t-test (two-tailed). Correlations between variables were determined by Spearman's correlation coefficient. Differences were considered significant at a $P$ value $<0.05$.

\section{RESULTS}

\section{$\mathrm{CD}^{+}{ }^{+} \mathrm{LOX}-1^{+}$PMN-MDSC frequency is increased in PB of NSCLC patients}

MDSCs that consist of PMN-MDSCs and M-MDSCs are immune suppressors in NSCLC. PMN-MDSCs have been identified as the major population of MDSCs in cancer patients, which induce the immune suppression in TME [25]. LOX-1 is a newly identified marker for distinguishing human PMN-MDSCs [29]. To compare the $\mathrm{CD} 15^{+} \mathrm{LOX}-1^{+}$PMN-MDSC frequency in NSCLC patients with that in healthy donors, we detected the proportion of $\mathrm{CD} 15^{+} \mathrm{LOX}-1^{+}$PMN-MDSCs in PB of the two groups. Detailed information of NSCLC patients were listed in Table 1 . Based on the results of FCM, it was indicated that CD15 ${ }^{+} \mathrm{LOX}-1^{+}$ PMN-MDSC frequency was significantly upregulated in the circulation of NSCLC patients $(20.84 \pm 1.342 \%)$ compared to healthy individuals $(3.215 \pm 0.1346 \%$ ) (Figure 1A, $P<0.0001)$. ARG1 is the main suppressor released by PMN-MDSCs, and we found that $A R G 1$ expression in PB cells increased significantly in NSCLC patients compared to health donors $(0.1679 \pm 0.0145$ vs $0.04951 \pm 0.0044)$ (Figure $1 \mathrm{~B}, P<0.0001)$. Moreover, the CD15 ${ }^{+} \mathrm{LOX}-1^{+}$PMN-MDSC frequency was positively correlated with the ARG1 expression in NSCLC patients $(\mathrm{r}=0.5951, P<0.0001)$ (Figure $1 \mathrm{C})$.

\section{$\mathrm{CD}^{+}{ }^{+} \mathrm{LOX}-1^{+}$PMN-MDSC is a potential biomarker for NSCLC diagnosis}

To confirm the potential of CD15+LOX-1+ PMN-MDSCs applied as a diagnostic marker for NSCLC, we firstly measured levels of CEA, CYFRA21-1 and CA125 in serum of NSCLC patients, and then analyzed their correlation with CD15 ${ }^{+} \mathrm{LOX}-1^{+} \mathrm{PMN}-\mathrm{MDSC}$ frequency. Results showed that levels of serum tumor markers including CEA $(7.348 \pm 1.024 \mathrm{ng} / \mathrm{mL})(P<0.0001)$, CYFRA21-1 $(7.436 \pm 0.9677 \mathrm{ng} / \mathrm{mL})(P<0.0001)$ and CA125 $(61.09 \pm 6.703 \mathrm{U} / \mathrm{mL})(P<0.0001)$ increased prominently in NSCLC patients compared with those in healthy donors (CEA: $1.77 \pm 0.1359 \mathrm{ng} / \mathrm{mL}$; CYFRA21-1: $1.799 \pm 0.1045 \mathrm{ng} / \mathrm{mL}$; CA125: $14.57 \pm 0.9361 \mathrm{U} / \mathrm{mL}$ ) (Figure 2A). We also found that $\mathrm{CD} 15^{+} \mathrm{LOX}-1^{+}$PMN-MDSC proportion was positively correlated with 
levels of CEA $(\mathrm{r}=0.6831, P=0.0021)$ and CYFRA21-1 $(\mathrm{r}=0.6598, P<0.0001)$, but not CA125 $(\mathrm{r}=0.2687, P$ $=0.0592$ ), in NSCLC patients (Figure 2B). Moreover, ROC curves of CD15 ${ }^{+} \mathrm{LOX}-1^{+}$PMN-MDSC frequency and tumor markers were analyzed. The results showed that AUC of PMN-MDSC frequency, CEA, CYFRA211 and CA125 in the diagnosis of NSCLC were 0.9996 (95\% CI: 0.9983-1.001), 0.8852 (95\% CI: 0.8226-0.9478), 0.9205 (95\% CI: 0.8670-0.9740) and 0.8209 (95\% CI: 0.7404-0.9015), respectively (Figure 2C). These results indicate that $\mathrm{CD} 15^{+} \mathrm{LOX}-1^{+}$PMN-MDSC is a potential biomarker for NSCLC diagnosis.

\section{The proportion of $\mathrm{CD}^{+}{ }^{+} \mathrm{LOX}-1^{+}$PMN-MDSCs is associated with NSCLC prognosis and recurrence after surgery}

To confirm the connection between CD $15^{+} \mathrm{LOX}-1^{+}$PMN-MDSCs and NSCLC prognosis, we detected the CD $15^{+}$LOX-1 ${ }^{+}$PMN-MDSC proportion in PB of NSCLC patients before and after surgical operation. The data showed that $\mathrm{CD} 15^{+} \mathrm{LOX}-1^{+}$PMN-MDSC frequency decreased significantly at 3 months after surgery $(20.84 \pm 1.3420$ vs $12.79 \pm 0.9396 \%, P=0.0058)$ (Figure $3 \mathrm{~A})$. Furthermore, we also measured the percentage of $\mathrm{CD} 15^{+} \mathrm{LOX}-1^{+}$PMN-MDSCs in NSCLC patients with or without recurrence after surgery. It was found that frequency of circulating $\mathrm{CD} 15^{+} \mathrm{LOX}-1^{+} \mathrm{PMN}-\mathrm{MDSCs}$ decreased significantly in NSCLC patients without recurrence compared to that in NSCLC patients with recurrence (13.76 \pm 0.9581 vs $19.91 \pm 0.7098$ $\%, P=0.0076$ ) (Figure 3B). In these cases, 11 samples could be traced from the NSCLC patients listed in

Table 1, in which there were 8 patients without recurrence and 3 patients with recurrence. By comparing the CD15 ${ }^{+}$LOX-1 ${ }^{+}$PMN-MDSC proportion in different groups, it was further confirmed that CD15+ $\mathrm{LOX}-1^{+}$ PMN-MDSCs were related to NSCLC recurrence after surgery $(9.944 \pm 1.569$ vs $21.25 \pm 0.7963 \%, P<0.0001)$ (Figure 3C). All these data confirm that circulating CD15+LOX-1 ${ }^{+}$PMN-MDSC frequency is associated with NSCLC prognosis and recurrence after surgery.

\section{DISCUSSION}

Lung cancer is the leading cause of cancer-related death worldwide. As the main histological subtype of lung cancer, NSCLC attracts a lot of attention. In the past 20 years, advantages in the diagnosis and treatment of NSCLC have been achieved tremendously, and the understanding for NSCLC biology and progression has also been more comprehensive [8]. For instance, applications of small molecule tyrosine kinase inhibitors and immunotherapy have resulted in unprecedented survival benefits in selected patients [33-36]. However, the overall cure and survival rates of NSCLC patients still remain at a low rate, especially in metastatic condition [8]. MDSCs that are a population of IMCs with immunosuppression are a main cause of NSCLC immune escape, metastasis and therapy resistance [37-40]. As the major population of MDSCs, PMN-MDSCs account for 70-80\% of total MDSCs in both tumor-bearing mice and cancer patients. PMN-MDSCs suppress anti-tumor immune responses by releasing high levels of arginase 1 (ARG1) and reactive oxygen species (ROS) [25]. The phenotype of PMN-MDSCs is complicated. PMN-MDSCs were previously characterized

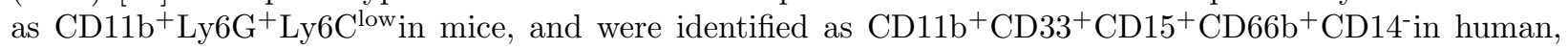
which shared the same phenotype with mature neutrophils [16]. LOX-1 is a newly defined marker that can distinguish human PMN-MDSCs from mature neutrophils, and peripheral LOX-1+ PMN-MDSCs is associated with anti-PD-1 therapy in NSCLC patients [29]. Here, we demonstrated that CD $15^{+}$LOX-1 $1^{+}$ PMN-MDSC proportion increased in PB of NSCLC patients. The frequency of circulating CD15 ${ }^{+} \mathrm{LOX}-1^{+}$ PMN-MDSCs was positively correlated with the levels of CEA and CYFRA21-1, and the combined detection of PMN-MDSCs and traditional tumor markers could enhance the diagnostic sensitivity and specificity for NSCLC. Moreover, we found that the proportion of CD $15^{+} \mathrm{LOX}-1^{+} \mathrm{PMN}-\mathrm{MDSCs}$ in PB of NSCLC patients decreased at 3 months after surgical resection, indicating that $\mathrm{CD} 15^{+} \mathrm{LOX}-1^{+} \mathrm{PMN}-\mathrm{MDSC}$ are associated with the prognosis of NSCLC. NSCLC recurrence after surgery is responsible for cancer associated mortalities after resection. To confirm the relationship between CD15+LOX-1+ PMN-MDSCs and NSCLC recurrence, we detected the PMN-MDSC frequency in NSCLC patients with or without recurrence after resection, and it was found that PMN-MDSC percentage was much higher in patients with recurrence. Therefore, CD15+LOX$1^{+}$PMN-MDSCs are associated with NSCLC recurrence after surgery. However, PB samples in this study were all from primary NSCLC patients in early stage that were diagnosed for the first time and had not received any treatment before. $\mathrm{CD} 15^{+} \mathrm{LOX}-1^{+}$PMN-MDSC presence in the circulation of III-IV NSCLC 
patients has not been revealed. Besides that, we only measured the frequency of $\mathrm{CD} 15^{+} \mathrm{LOX}-1^{+} \mathrm{PMN}-$ MDSCs before/after surgical treatment, but the relationship between CD15+ LOX-1 ${ }^{+}$PMN-MDSCs and other treatments, such as radiotherapy, chemotherapy and targeted therapy, still needs further investigations.

\section{CONCLUSION}

In summary, we identified that CD15 ${ }^{+} \mathrm{LOX}-1^{+}$PMN-MDSC proportion increases in PB of NSCLC patients, and combined detection of PMN-MDSCs and traditional tumor markers can promote the diagnostic sensitivity and specificity for NSCLC. Moreover, CD $15^{+}$LOX-1 $1^{+}$PMN-MDSCs are associated with NSCLC prognosis and recurrence after surgery.

\section{ACKNOWLEDGEMENTS}

This work was supported by Jiangsu Planned Projects for Postdoctoral Research Funds (2018K253C), China's Post-doctoral Science Fund (2018ZM642225), and Funds of Nanjing Medical University Affiliated Cancer Hospital (ZM201704).

\section{DECLARATION OF INTEREST}

No conflicts of interest.

\section{AUTHOR CONTRIBUTION:}

Conception: Xinyu Tian, Ting Wang

Interpretation or analysis of data: Yue Tao, Qisi Zheng

Preparation of the manuscript: Ting Wang, Han Shen

Revision for important intellectual concept: Ting Wang

Supervision: Ting Wang, Han Shen

All authors approved the final version.

\section{REFERENCES}

1. Siegel RL, Miller KD, Jemal A. Cancer statistics, 2015. CA Cancer J Clin . 2015;65:5-29.

2. Torre LA, Bray F, Siegel RL, Ferlay J, Lortet-Tieulent J, Jemal A. Global cancer statistics, 2012. CA Cancer J Clin . 2015;65:87-108.

3. Oser MG, Niederst MJ, Sequist LV, Engelman JA. Transformation from non-small-cell lung cancer to small-cell lung cancer: molecular drivers and cells of origin. Lancet Oncol . 2015;16:e165-172.

4. Testa U, Castelli G, Pelosi E. Lung Cancers: Molecular Characterization, Clonal Heterogeneity and Evolution, and Cancer Stem Cells. Cancers (Basel) . 2018;10.

5. Molina JR, Yang P, Cassivi SD, Schild SE, Adjei AA. Non-small cell lung cancer: epidemiology, risk factors, treatment, and survivorship. Mayo Clin Proc. 2008;8:584-594.

6. Hirsch FR, Scagliotti GV, Mulshine JL, et al. Lung cancer: current therapies and new targeted treatments. Lancet . 2017;389:299-311.

7. Zugazagoitia J, Guedes C, Ponce S, Ferrer I, Molina-Pinelo S, Paz-Ares L. Current Challenges in Cancer Treatment. Clin Ther . 2016;38:1551-1566.

8. Herbst RS, Morgensztern D, Boshoff C. The biology and management of non-small cell lung cancer. Nature . 2018;553:446-454.

9. Liu T, Song Z, Gai Y. Circular RNA circ_0001649 acts as a prognostic biomarker and inhibits NSCLC progression via sponging miR-331-3p and miR-338-5p. Biochem Biophys Res Commun. 2018; 50 , 1503-1509.

10. Pignon JP, Tribodet H, Scagliotti GV, et al. Lung adjuvant cisplatin evaluation: a pooled analysis by the LACE Collaborative Group. J Clin Oncol . 2008;26:3552-3559. 
11. Kris MG, Gaspar LE, Chaft JE, et al. Adjuvant Systemic Therapy and Adjuvant Radiation Therapy for Stage I to IIIA Completely Resected Non-Small-Cell Lung Cancers: American Society of Clinical Oncology/Cancer Care Ontario Clinical Practice Guideline Update.J Clin Oncol . 2017;35:2960-2974.

12. Lu Z, Zou J, Li S, et al. Epigenetic therapy inhibits metastases by disrupting premetastatic niches. Nature . 2020;579:284-290.

13. Iams WT, Porter J, Horn L. Immunotherapeutic approaches for small-cell lung cancer. Nat Rev Clin Oncol . 2020;17:300-312.

14. Forde PM, Chaft JE, Smith KN, et al. Neoadjuvant PD-1 Blockade in Resectable Lung Cancer. $N$ Engl J Med . 2018;378:1976-1786.

15. Postmus PE, Kerr KM, Oudkerk M, et al. Early and locally advanced non-small-cell lung cancer (NSCLC): ESMO Clinical Practice Guidelines for diagnosis, treatment and follow-up. Ann Oncol . 2017;28:iv1-iv21.

16. Tian X, Shen H, Li Z, Wang T, Wang S. Tumor-derived exosomes, myeloid-derived suppressor cells, and tumor microenvironment. J Hematol Oncol . 2019;12:84.

17.Deng Z, Rong Y, Teng Y, et al. Exosomes miR-126a released from MDSC induced by DOX treatment promotes lung metastasis. Oncogene . 2017;36:639-651.

18. Bremnes RM, Busund LT, Kilvaer TL, et al. The Role of Tumor-Infiltrating Lymphocytes in Development, Progression, and Prognosis of Non-Small Cell Lung Cancer. J Thorac Oncol . 2016;11:789-800.

19. Caetano MS, Zhang H, Cumpian AM, et al. IL6 Blockade Reprograms the Lung Tumor Microenvironment to Limit the Development and Progression of K-ras-Mutant Lung Cancer. Cancer Res . 2016;76:3189-3199.

20. Gabrilovich DI, Nagaraj S. Myeloid-derived suppressor cells as regulators of the immune system. Nat Rev Immunol . 2009;9:162-174.

21. Bruger AM, Dorhoi A, Esendagli G, et al. How to measure the immunosuppressive activity of MDSC: assays, problems and potential solutions. Cancer Immunol Immunother . 2019;68:631-644.

22. Gabrilovich DI. Myeloid-Derived Suppressor Cells.Cancer Immunol Res . 2017;5:3-8.

23. Ostrand-Rosenberg S. Myeloid derived-suppressor cells: their role in cancer and obesity. Curr Opin Immunol . 2018;51:68-75.

24. Kumar V, Patel S, Tcyganov E, Gabrilovich DI. The Nature of Myeloid-Derived Suppressor Cells in the Tumor Microenvironment. Trends Immunol . 2016;37:208-220.

25. Tian X, Zheng Y, Yin K, et al. LncRNA AK036396 Inhibits Maturation and Accelerates Immunosuppression of Polymorphonuclear Myeloid-Derived Suppressor Cells by Enhancing the Stability of Ficolin B. Cancer Immunol Res . 2020;8(4):565-577.

26. Barrera L, Montes-Servin E, Hernandez-Martinez JM, et al. Levels of peripheral blood polymorphonuclear myeloid-derived suppressor cells and selected cytokines are potentially prognostic of disease progression for patients with non-small cell lung cancer. Cancer Immunol Immunother . 2018;67:1393-1406.

27. de Goeje PL, Bezemer K, Heuvers ME, et al. Immunoglobulin-like transcript 3 is expressed by myeloid-derived suppressor cells and correlates with survival in patients with non-small cell lung cancer.Oncoimmunology . 2015;4:e1014242.

28. Zhang Z, Huang X, Wang E, Huang Y, Yang R. Suppression of Mll1-Complex by Stat3/CebpbetaInduced miR-21a/21b/181b Maintains the Accumulation, Homeostasis, and Immunosuppressive Function of Polymorphonuclear Myeloid-Derived Suppressor Cells. J Immunol . 2020;204:3400-3415.

29. Condamine T, Dominguez GA, Youn JI, et al. Lectin-type oxidized LDL receptor-1 distinguishes population of human polymorphonuclear myeloid-derived suppressor cells in cancer patients. Sci Immunol . $2016 ; 1$. 
30. Nan J, Xing YF, Hu B, et al. Endoplasmic reticulum stress induced LOX-1(+) CD15(+) polymorphonuclear myeloid-derived suppressor cells in hepatocellular carcinoma. Immunology . 2018;154:144-155.

31. Veglia F, Perego M, Gabrilovich D. Myeloid-derived suppressor cells coming of age. Nat Immunol . 2018;19:108-119.

32. Chai E, Zhang L, Li C.LOX-1+ PMN-MDSC enhances immune suppression which promotes glioblastoma multiforme progression. Cancer Manag Res. 2019; 11, 7307-7315.

33. Singh M, Jadhav HR. Targeting non-small cell lung cancer with small-molecule EGFR tyrosine kinase inhibitors. Drug Discov Today . 2018;23:745-753.

34. Cascone T, Morelli MP, Ciardiello F. Small molecule epidermal growth factor receptor (EGFR) tyrosine kinase inhibitors in non-small cell lung cancer. Ann Oncol . 2006;17:ii46-8.

35. Suresh K, Naidoo J, Lin CT, Danoff S. Immune Checkpoint Immunotherapy for Non-Small Cell Lung Cancer: Benefits and Pulmonary Toxicities. Chest . 2018;154:1416-1423.

36. Osmani L, Askin F, Gabrielson E, Li QK. Current WHO guidelines and the critical role of immunohistochemical markers in the subclassification of non-small cell lung carcinoma (NSCLC): Moving from targeted therapy to immunotherapy. Semin Cancer Biol . 2018;52:103-109.

37. Dajon M, Iribarren K, Petitprez F, et al. Toll like receptor 7 expressed by malignant cells promotes tumor progression and metastasis through the recruitment of myeloid derived suppressor cells. Oncoimmunology . 2019;8:e1505174.

38. Li YD, Lamano JB, Lamano JB, et al. Tumor-induced peripheral immunosuppression promotes brain metastasis in patients with non-small cell lung cancer. Cancer Immunol Immunother . 2019;68:1501-1513.

39. Zheng Y, Tian X, Wang T, et al. Long noncoding RNA Pvt1 regulates the immunosuppression activity of granulocytic myeloid-derived suppressor cells in tumor-bearing mice. Mol cancer . 2019;18:61.

40. Weber R, Fleming V, Hu X, et al. Myeloid-Derived Suppressor Cells Hinder the Anti-Cancer Activity of Immune Checkpoint Inhibitors. Front Immunol . 2018;9:1310.

Table 1. Clinical demographic characteristics of NSCLC subjects $(\mathrm{n}=50)$

\begin{tabular}{|c|c|c|c|c|c|c|c|}
\hline Parameter & Number & Number & Number & Number & $\begin{array}{l}\text { CD15+LOX- } \\
1^{+} \\
\text {PMN- } \\
\text { MDSC } \\
\text { frequency } \\
(\%) \\
{[?] 20}\end{array}$ & $\begin{array}{l}\text { CD15+LOX- } \\
1^{+} \\
\text {PMN- } \\
\text { MDSC } \\
\text { frequency } \\
(\%) \\
<20\end{array}$ & $\begin{array}{l}\mathrm{CD} 15^{+} \mathrm{LOX}- \\
1^{+} \\
\text {PMN- } \\
\text { MDSC } \\
\text { frequency } \\
(\%) \\
P \text { value } \\
(\text { Mean } \pm \mathrm{SD})\end{array}$ \\
\hline Age & & & & & & & 0.9757 \\
\hline$<60$ & 14 & 14 & 14 & 14 & 5 & 9 & \\
\hline [?]60 & 36 & 36 & 36 & 36 & 15 & 21 & \\
\hline Gender & & & & & & & 0.0176 \\
\hline Male & 29 & 29 & 29 & 29 & 13 & 16 & \\
\hline Female & 21 & 21 & 21 & 21 & 7 & 14 & \\
\hline $\begin{array}{l}\text { Tumor } \\
\text { size }\end{array}$ & & & & & & & \\
\hline$[?] 3 \mathrm{~cm}$ & 50 & 50 & 50 & 50 & 20 & 30 & \\
\hline$>3 \mathrm{~cm}$ & 0 & 0 & 0 & 0 & 0 & 0 & \\
\hline $\begin{array}{l}\text { Smoking } \\
\text { history }\end{array}$ & & & & & & & 0.3784 \\
\hline
\end{tabular}




\begin{tabular}{|c|c|c|c|c|c|c|c|}
\hline Smokers & 26 & 26 & 26 & 26 & 9 & 17 & \\
\hline $\begin{array}{l}\text { Never } \\
\text { smokers }\end{array}$ & 24 & 24 & 24 & 24 & 11 & 13 & \\
\hline $\begin{array}{l}\text { Lymph } \\
\text { node } \\
\text { metastasis }\end{array}$ & $\begin{array}{l}\text { Lymph } \\
\text { node } \\
\text { metastasis }\end{array}$ & & & & & & \\
\hline Positive & 0 & 0 & 0 & 0 & 0 & 0 & \\
\hline Negative & 50 & 50 & 50 & 50 & 20 & 30 & \multirow[b]{2}{*}{0.4653} \\
\hline $\begin{array}{l}\text { TNM } \\
\text { stage }\end{array}$ & & & & & & & \\
\hline I & 33 & 33 & 33 & 33 & 10 & 23 & \multirow{5}{*}{0.0832} \\
\hline II & 14 & 14 & 14 & 14 & 10 & 4 & \\
\hline $\begin{array}{l}\text { Histological } \\
\text { tumor } \\
\text { type }\end{array}$ & $\begin{array}{l}\text { Histological } \\
\text { tumor } \\
\text { type }\end{array}$ & & & & & & \\
\hline $\begin{array}{l}\text { Squamous } \\
\text { cell } \\
\text { carcinoma }\end{array}$ & $\begin{array}{l}\text { Squamous } \\
\text { cell } \\
\text { carcinoma }\end{array}$ & $\begin{array}{l}\text { Squamous } \\
\text { cell } \\
\text { carcinoma }\end{array}$ & $\begin{array}{l}\text { Squamous } \\
\text { cell } \\
\text { carcinoma }\end{array}$ & 23 & 12 & 11 & \\
\hline Adenocarcin & Adenocarcin & Addenocarc & 187 & 27 & 8 & 19 & \\
\hline
\end{tabular}

Figure legends:

Figure 1. CD15 ${ }^{+}$LOX-1 ${ }^{+}$PMN-MDSC frequency in the circulation of healthy donors and NSCLC patients.

The frequency of circulating CD $15^{+} \mathrm{LOX}-1^{+}$PMN-MDSCs in healthy donors $(\mathrm{n}=50)$ and NSCLC patients $(\mathrm{n}=50)$ were measured by FCM. (B) $A R G 1$ expression was detected in PB of healthy donors $(\mathrm{n}=50)$ and NSCLC patients $(\mathrm{n}=50)$. (C) The correlation analysis between CD $15^{+} \mathrm{LOX}-1^{+}$PMN-MDSC frequency and $A R G 1$ expression in NSCLC patients $(\mathrm{n}=50)$. ${ }^{* * *} P<0.001$.

Figure 2. The potential application of $\mathrm{CD} 15^{+} \mathrm{LOX}-1^{+}$PMN-MDSCs in NSCLC diagnosis.

(A) Levels of CEA, CYFRA21-1, and CA125 in serum of NSCLC patients were measured ( $\mathrm{n}=50)$. (B) The correlation of $\mathrm{CD} 15^{+} \mathrm{LOX}-1^{+}$PMN-MDSC frequency with levels of tumor markers. (C) ROC curves. ${ }^{* * *} P$ $<0.001$.

Figure 3. Circulating CD15+LOX-1 ${ }^{+}$PMN-MDSCs are associated with NSCLC prognosis and recurrence after surgery.

(A) $\mathrm{CD} 15^{+}$LOX-1+ PMN-MDSC frequency in PB of NSCLC patients before $(\mathrm{n}=50)$ or after surgical resection $(\mathrm{n}=50)$. (B) $\mathrm{CD} 15^{+} \mathrm{LOX}-1^{+}$PMN-MDSC frequency in PB of NSCLC patients with $(\mathrm{n}=50)$ or without $(\mathrm{n}=50)$ recurrence post-surgery. $(\mathrm{C}) \mathrm{CD} 15^{+} \mathrm{LOX}-1^{+}$PMN-MDSC frequency was compared in traced NSCLC patients with $(\mathrm{n}=8)$ or without $(\mathrm{n}=3)$ recurrence post-surgery. ${ }^{* *} P<0.01,{ }^{* * *} P<0.001$. 

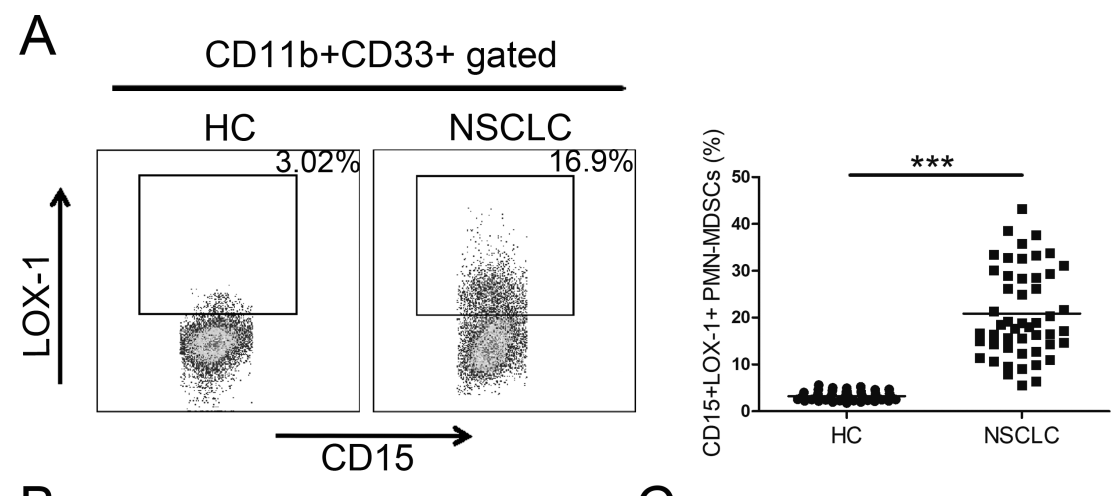

B

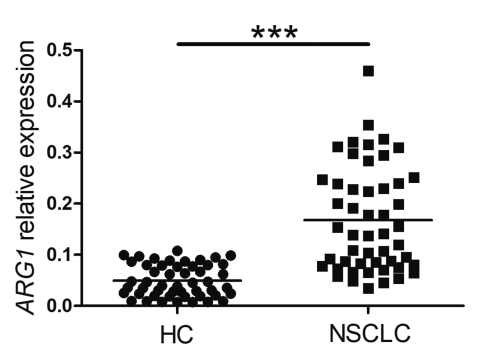

C

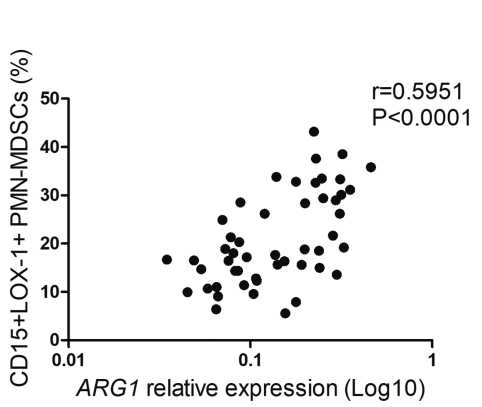

A

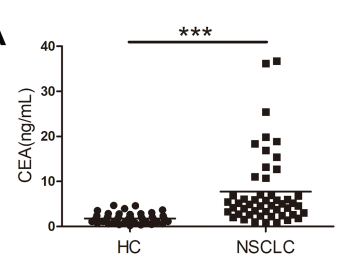

B

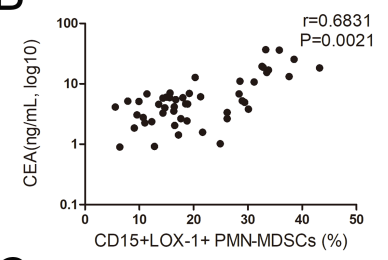

C
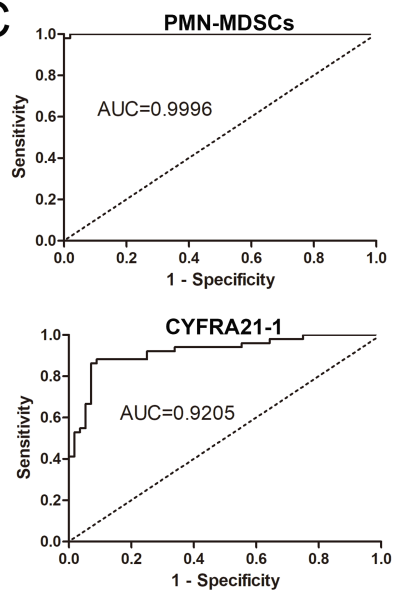
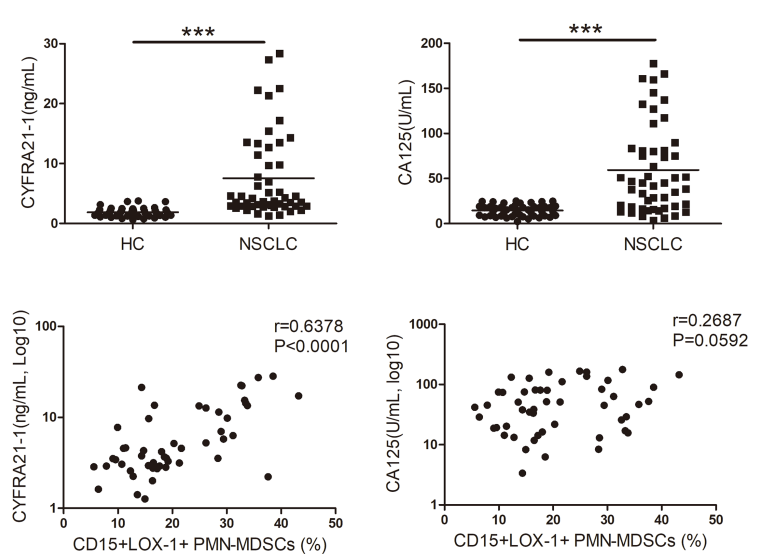

- Sensitivity
.... Identity

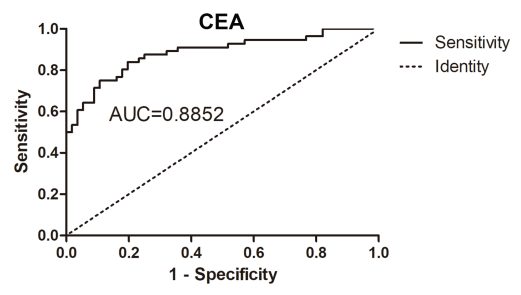

- Sensitivity
.... Identity

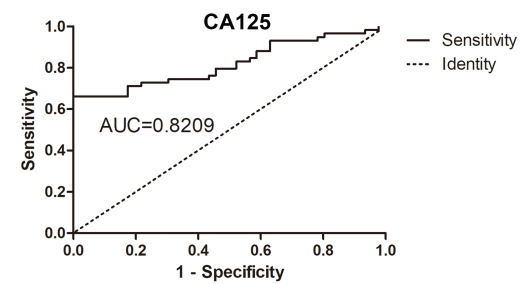


A CD11b+CD33+ gated
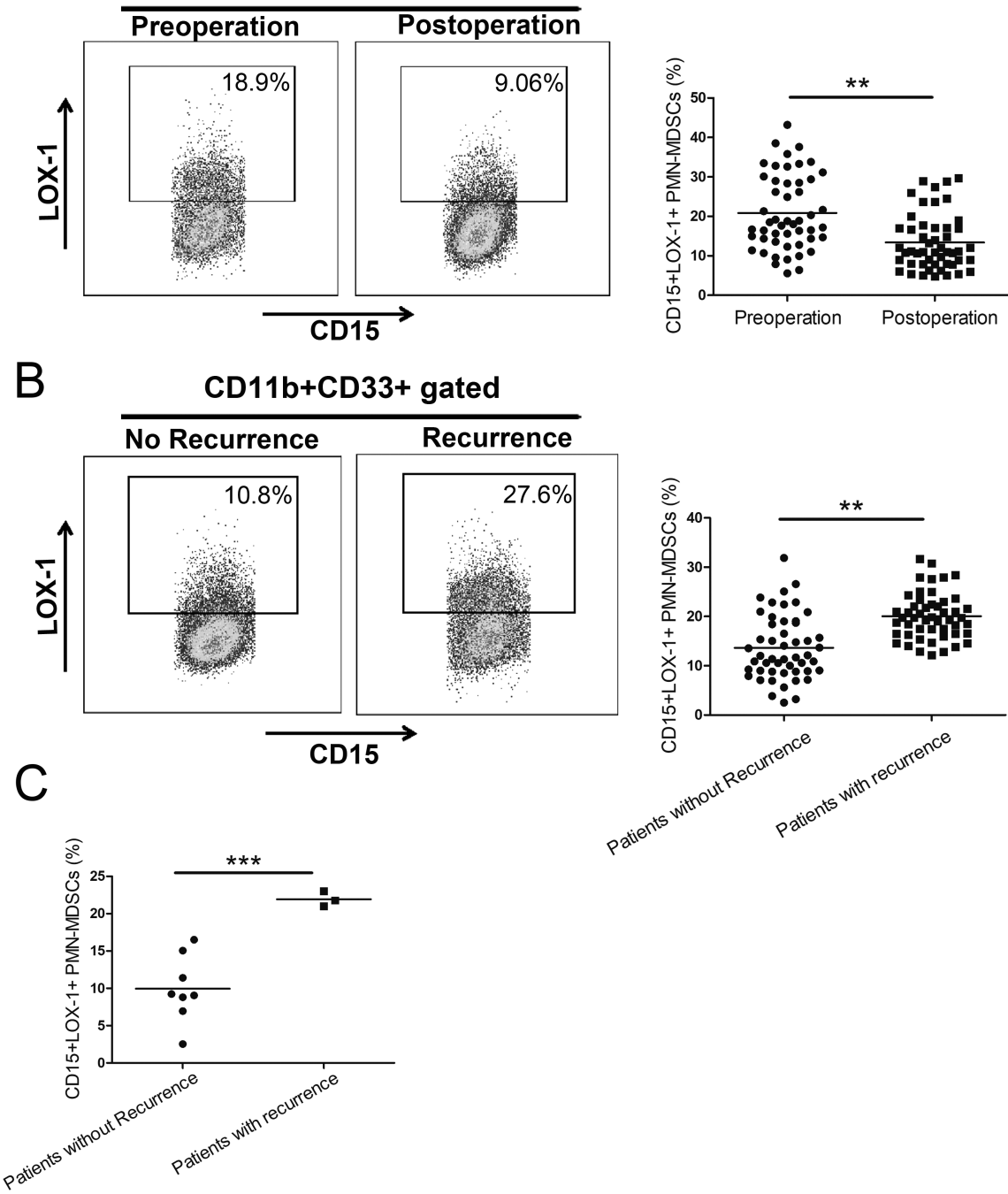